\title{
Novel "crowbar effect" approach to improve success rate of recanalization of coronary chronic total occlusions
}

\author{
Ruifang Liu ${ }^{\mathrm{a}}$, Fangxing $\mathrm{Xu}^{\mathrm{a}}$ and Tongku Liu ${ }^{\mathrm{b}, *}$ \\ ${ }^{a}$ Beijing Anzhen Hospital, The Capital Medical University, Beijing, China \\ ${ }^{\mathrm{b}}$ Affiliated Hospital of Beihua University, Jilin, Jilin, China
}

\begin{abstract}
.
BACKGROUND: Coronary chronic total occlusions (CTO) are the most challenging lesions to treat percutaneously. Thus, consistent efforts are made to develop new approaches to treat CTO.

OBJECTIVE: To explore the key points of a novel "crowbar effect" approach to improve the success rate of recanalization of CTOs.

METHODS: One hundred and fifty-seven patients with CTO were treated with PCI using the regular antegrade guide wire approach. Of them, 36 patients $(22.9 \%)$ showed that while the first guide wire was inserted into the CTO lesions, a small balloon had difficulty passing through the CTO lesions. For those patients, the new crowbar effect technique was used to allow the balloon to pass through the lesions.

RESULTS: The coronary CTO vessels in 35 patients $(97.2 \%)$ were completely opened. Coronary perforation occurred in 5 patients $(13.8 \%)$. This perforation was properly treated and did not lead to serious complications.

CONCLUSION: The crowbar effect technique proved successful as an alternative antegrade method for opening CTO. The procedure of this novel method is easy to accomplish and success rates are high.
\end{abstract}

Keywords: Coronary artery, chronic total occlusion, percutaneous coronary intervention, crowbar effect technique, success rate [1]

\section{Introduction}

Coronary chronic total occlusion (CTO) lesions refer to coronary artery occlusions that are present for at least three months. The benefits of CTO recanalization include symptom relief, improved regional and global left ventricular function, improved quality of life, and better long-term survival rate versus failed recanalization CTO treatments with percutaneous coronary intervention (PCI) [1-3]. Successful revascularization may improve angina, increase exercise capacity, reduce the possibility of the late coronary artery bypass graft (CABG) surgery [4], and improve quality of life. CTO lesions are typically complex, and associated with atheromatous plaque, thrombosis, and fiber endangium proliferation. The recanalization of CTO lesions is one of the most challenging PCI procedures [4-6] and has been called "the last frontier" of PCI. The primary reasons for this are associated with the technical complexity, poor

\footnotetext{
${ }^{*}$ Corresponding author: Tongku Liu, Affiliated Hospital of Beihua University, Jilin, Jilin, China. E-mail: liutongku2010 @163.com.
}

0928-7329/15/\$35.00 (C) 2015 - IOS Press and the authors. All rights reserved

This article is published online with Open Access and distributed under the terms of the Creative Commons Attribution NonCommercial License. 
success rate of PCI operations, and adverse acute procedure-related complications, as well as increased radiation exposure [7]. PCI of CTOs is a rapidly developing field [8[9]. Both antegrade and retrograde approaches have been used in PCI to open CTO lesions (CTO-PCI) [10], and the relatively low success rate is a major concern for most physicians. To achieve a successful PCI with either of these methods, the key is to put a small balloon through the CTO lesions following the guide wires. The present study sought to establish a novel alternative approach by applying the "crowbar effect" to assist the balloon pass through the CTO lesion and thus improve the success rate of CTO recanalization.

\section{Methods}

\subsection{Patients selected}

A total of 157 patients with coronary CTO lesions were treated with percutaneous coronary intervention (PCI) via regular antegrade guide wire approaches from January 2010 to October 2014. Of them, 36 patients $(22.9 \%)$ with CTO lesions showed that although the first guide wire was inserted into the CTO lesions, which was confirmed in the true lumen by using multi-angle coronary angiography (CAG), a small balloon had difficulty passing through the CTO lesions. For those cases, the new crowbar effect technique was used to allow the small balloon to pass through the lesions, improving the success rate of recanalization of the CTO. Out of the 36 subjects, there were 25 males (69.4\%) and 11 females $(30.6 \%)$. The ages of the patients were between 41 and 82 years old (mean age was $65 \pm 7$ years). All of these patients suffered from unstable angina pectoris [11] (CCS angina class 3-4 for at least 3 months). In the 36 cases, a history of myocardial infarction (MI) was documented in 32 cases (88.9\%). According to each patient's history of angina pectoris, the duration of coronary artery occlusion in the patients without a history of MI was doped out. The $Q$ waves corresponding to the CTO artery territory was confirmed by electrocardiographic evidence in 28 cases $(77.8 \%)$. A total of 31 cases $(86.1 \%)$ with CTO showed relatively normal left ventricular function defined by $\mathrm{EF} \geqslant 50 \%$.

\subsection{Coronary artery angiography characteristics of patients}

Coronary artery angiography (CAG) characteristics seen in the 36 cases with CTO lesions included: 1 ) The presence of a tapered stump or funnel-shaped occlusion in the occluded segment; 2) Only a few or no capillary side branches or intracoronary "bridging" collaterals at the proximal end of the occlusion; 3) Occluded vessels with an optional puncturable point; 4) Distal segment of the occluded vessel with good collateral circulation on retrograde perfusion imaging; and 5) Occluded vessel segments that were relatively straight without severe calcification and lengths of $20 \mathrm{~mm}-45 \mathrm{~mm}$ (15 patients had occluded vessels at lengths of $20 \mathrm{~mm}-30 \mathrm{~mm}, 17$ patients with lengths of $31 \mathrm{~mm}-40 \mathrm{~mm}$, and 4 patients with lengths of $40 \mathrm{~mm}-45 \mathrm{~mm}$ ). In addition, all patients had 0 grade blood flow of thrombolysis in myocardial infarction (TIMI) in the occluded vessel segments, and 29 subjects (80.6\%) showed collateral circulation on their retrograde perfusion imaging.

\subsection{PCI procedure}

\subsubsection{Preoperative and intraoperative using drugs}

All patients were routinely administrated $300 \mathrm{mg}$ aspirin and $300 \mathrm{mg}$ clopidogrel before PCI and aspirin $100 \mathrm{mg} /$ day and clopidogrel $75 \mathrm{mg} /$ day for 12 months after the procedure. A bolus of heparin $(100 \mathrm{u} / \mathrm{Kg})$ was injected immediately before the procedure followed by intravenous infusion of 
$1000 \mathrm{u}$ /hour to maintain an activated clotting time $>250$ seconds during the procedure. A subcutaneous injection of heparin $5000 \mathrm{U}$ twice daily was administrated for three days after the procedure. As needed, nitroglycerin ( $200 \mathrm{ug}-400 \mathrm{ug}$ ) was injected into the intra-coronary artery during the procedure.

\subsubsection{Selection of guiding catheter and approach}

The PCI procedures were performed via the standard femoral, 5 cases (13.9\%), and right radial artery, 31 cases $(86.1 \%)$, approach. XB, BL, or EBU guiding catheters were used for the left coronary arteries (LCA) and JR or AL guiding catheters for the right coronary arteries (RCA). When the guiding catheter support was insufficient, a. 035-inch guide wire for CAG (the guide wire was backwards to the insert) was inserted into the guiding catheter as far as the first bend of the catheter in order to increase the guiding catheter's support strength.

\subsubsection{Selection of guide wires and operation}

Selection of guide wires was mainly according to the features of the CTO lesion. Guide wire with non-hydrophilic coating and moderate stiffness was preferred. Under the support of a micro catheter or a small balloon $(1.25 \mathrm{~mm} \times 15 \mathrm{~mm})$, the wire was used to puncture the fiber cap of the proximal segment of the CTO lesion to pass through into the distal vessel segment. Using multi-angle CAG, the guide wire was confirmed to be in the true lumen and the balloon was pushed to pass the CTO lesion. If it was difficult for the balloon to pass, a second, relatively stiffer guide wire with hydrophilic coating was inserted along the first wire trace through the CTO lesion. After taking out one of the wires, the balloon was sent again into the lesion. If that also failed, another soft guide wire was inserted along the previous wire trace followed by the insertion of a third, stiff wire in the same way. Finally, the two stiff wires were removed and it was attempted to insert a small balloon $(1.25 \mathrm{~mm} \times 15 \mathrm{~mm})$ through the CTO lesions with the remaining soft guide wire. If the balloon was still unable to enter the lesions, two guide wires (one soft and one moderately stiff) were inserted again as described above. The small balloon was again inserted and was repeatedly inflated with high pressure (12 atm-16 atm) and with push balloon power. The small balloon was pushed forward $2 \mathrm{~mm}-3 \mathrm{~mm}$ with each inflation. This repetitive inflation eventually moved the balloon through the lesions into the distal vessel. After withdrawing two wires, a larger balloon (usually $2.0 \mathrm{~mm} \times 20 \mathrm{~mm}$ ) was inserted along the only remaining soft guide wire, and pre-dilatation was completed by inflation followed by the drug-eluting stent (DES) implantation. If the small balloon failed to pass through the lesions after repeated attempts, the PCI was terminated.

\subsubsection{Success criteria of CTO-PCI}

A successful PCI is defined as a successful guide wire insertion, balloon dilatation, and stent implantation. It is also characterized by a $<20 \%$ residual stenosis and TIMI 3 grade of the target vessel, as well as without in-hospital major adverse cardiac events (MACEs), including cardiac death, MI with new Qwaves on electrocardiogram (ECG), emergency revascularization of target vessel (including repeat PCI and CABG surgery), or non-Q-wave MI ( $>2$ times elevated creatine kinase-MB or $>3$ times elevated troponin I compared with the upper limit of normal, and with recurrent ischemic symptoms following PCI).

\section{Statistical analysis}

All statistical calculations were performed using Statistical Package for the Social Sciences (SPSS Statistics) 17.0. The data are expressed as mean \pm standard deviation $(\bar{x} \pm s)$ for continuous variables. Categorical data are expressed as percentages. 


\section{Results}

\subsection{Baseline characteristics}

In 36 patients, there were 25 males and 11 females. The mean age was $(65 \pm 7)$ years. Among those, 32 patients $(88.9 \%)$ had history of MIs. Occlusive coronary vascular distribution in patients with CTO lesions included right coronary artery (RCA) 22 cases $(61.1 \%)$, left anterior descending artery (LAD) 5 cases (13.9\%), and left circumflex branch artery (LCX) 9 cases (25.0\%). Occlusive duration of CTO was 36.9 months \pm 26.1 months. A total of 29 cases (80.6\%) had collateral circulation by CAG images.

\subsection{Success rate of PCI and coronary perforation rate}

The success rate of PCI operations using the crowbar effect was improved. In 36 patients with CTO lesions, 35 were completely opened with the crowbar effect technique in the absence of a special method, such as, using tornus micro catheter, balloon anchoring technology and so on. The success rate of CTO recanalization was $97.2 \%$. One case $(2.8 \%)$ failed because the guide wire was accidentally inserted into the dissection of a blood vessel and there was no blood flow recovery after balloon dilatation. Coronary artery perforation happened in 5 patients $(13.8 \%)$ because the guide wire punctured the vessel wall during the procedure. Although there was a slight increase of the rate of coronary perforation, all coronary perforations were properly treated and did not lead to serious complications. Besides coronary perforation, no other complications were observed during the procedures. Major adverse cardiac events (MACE) in-hospital (including cardiac death, urgent bypass surgery, Q-wave MI, or target vessel revascularization) did not occur in any of the researched cases.

\subsection{Implanted stents character in the PCI successful cases}

Drug-eluting stents (DESs) were implanted in the successful PCI cases. The number of implanted stents was $(\bar{x} \pm S) 1.31 \pm 0.45$. The lengths of the implanted stents were $(\bar{x} \pm S) 25.70 \mathrm{~mm} \pm 5.37 \mathrm{~mm}$. Diameters of the implanted stents were $(\bar{x} \pm S) 3.06 \mathrm{~mm} \pm 0.25 \mathrm{~mm}$.

\section{Discussion}

PCI for CTO recanalization is a high-risk procedure, but when successful, the patients enjoy longterm benefits. A successful recanalization of CTO lesions may improve all-cause mortality and have lower rates of MACE [12]. There are many ways to perform CTO recanalization, but the most popular technique is the conventional method using antegrade guide wires [13], which is mainly intended for easy to intermediate and very difficult lesions. For this reason, a simple antegrade guide wire step-up approach with the new generation of wires might result in reduced failure rates in easy, intermediate, and very difficult CTO lesions. This is the first report on the antegrade crowbar effect technique being used to open CTO lesions. The results indicated that this new approach significantly improved CTOPCI success rates without increasing serious complications. Among the 36 patients with CTOs, 35 cases were successful, and the success rate of CTO recanalization was $97.2 \%$. Compared to retrograde CTOPCI, the antegrade CTO-PCI was associated with a lower incidence of target lesion revascularization (TLR) [14]. The crowbar effect technique is an alternative antegrade method for effectively opening CTO lesions. The procedure of this novel method is easy to operate and achieves high success rates, 
especially when the support strength of the guiding catheter is not enough. Compared to the reverse technology of the retrograde guide wire approach, this method is simpler, the operation time is shorter, $\mathrm{X}$-ray exposure time is less, dosage of contrast agent is less, and it is a smart choice in the absence of a special method, especially when the support of the guiding catheter is insufficient.

\subsection{The novelty of "crowbar effect" technique}

The benefits of CTO recanalization include lessening of symptoms, improvement in left ventricular (LV) function, and survival. With the improvements in guide wires, the success rates of CTO-PCI have increased to $70 \%$ and higher. However, this success rate is still far below that of PCI in non-CTO lesions. The characteristics of CTOs, such as fibrotic shell, calcific atherosclerotic plaque, and relatively longer lengths of lesions, make CTO-PCI the most challenging coronary interventional practice. After even successful guide wire passing CTO lesions, the difficulty of inserting a balloon is usually the cause of failure of the CTO-PCI. Here, a novel method was introduced to significantly improve the balloon passage. When there is difficulty in inserting the balloon into the lesions, another two "assistant" guide wires are inserted along the pre-inserted guide wire. Relaying these wires, the small balloon gains extra pushing force with normal pressure (12 atm-16 atm) as previously described in detail. In most cases, this crowbar effect can allow the balloon to pass through the CTO lesion.

\subsection{The key points of successful "crowbar" procedure}

When utilizing the crowbar effect approach, critical procedures for success include the following steps: 1) Insert a guide wire through the CTO lesion; 2) Insert second and third wires along the trace of the first wire into the true lumen of the distal vessel segment; 3) Send a small balloon with a diameter of $1.25 \mathrm{~mm}$ to $1.50 \mathrm{~mm}$ into the lesions by repeatedly inflating the balloon with high pressure (14 atm$16 \mathrm{~atm}$ ), which moves the balloon forward $2 \mathrm{~mm}-3 \mathrm{~mm}$ with each inflation until it passes through the occlusive vascular segment; 4) Withdraw the second and third guide wires and insert a bigger balloon with a diameter of $2.0 \mathrm{~mm}$ or larger along the retained soft guide wire; 5) Enlarge the channel by inflating the balloon and implant a DES.

\subsection{The indications for applying the "crowbar effect" approach to open CTO lesions}

The indication for the crowbar effect approach is when guide wires can cross CTO lesions, but a balloon can't pass through, especially when the support of the guiding catheter is insufficient.

\subsection{About the success rate of $C T O$ recanalization}

The success rate of CTO-PCI is associated with the expert operators, technique, and selection criteria of the CTO lesions. Highly experienced CTO-operators use a predominance of favorable characteristics of CTO lesions to achieve success rates above $90 \%, 15$. The success rate may drop to below $60 \%-70 \%$ in the presence of one or more unfavorable predictive factors. Indications of CTO-PCI in this study were identified as medically refractory angina, large area (over 10\% of the total myocardium) of ischemia by noninvasive studies, and favorable angiographic appearance defined as a vessel diameter of CTO $>2.5 \mathrm{~mm}$, the length of occlusion segment being $10 \mathrm{~mm}-45 \mathrm{~mm}$ with imaging evaluation, tapered proximal CTO cap, proximal vessel angulation of $<45^{\circ}$, and single or double total occlusions within the target vessel and without the presence of bridging collaterals. The success rate of recanalization using 
the crowbar effect technique was significantly improved for the described CTO lesions. Karmpaliotis et al. [16] reported the success rate of CTO-PCI as $85.6 \%$ (137/160). Han et al. [17] reported that 1,147 out of 1,263 cases $(90.8 \%)$ with CTO in a single center registered PCI study were successfully recanalized, which was the highest success rate in registered research. The report of the ERCTO registry by the EuroCTO club showed a high procedural success rate (82.0\%) obtained by expert European operators [18]. The study conducted by Morino et al. [19] reported a success rate (86.6\%) of CTO-PCI in the J-CTO registry (multicenter CTO registry in Japan). Fefer et al. [20] reported the success rate of CTO-PCI from a Canadian multicenter chronic total occlusions registry was $70.0 \%$. Multiple papers showed the success rates of CTO-PCI as being between 50-70\% [21-23]. Tesfaldet et al. [24] reported that antegrade wire escalation, antegrade dissection/reentry, and retrograde crossing were utilized in $97.2 \%, 46.6 \%$, and $46.6 \%$ of cases, respectively. Among successful cases, the CTO crossing technique was the antegrade guide wire escalation. Christopoulos et al. [25] reported on the application of a hybrid approach to chronic total occlusions in patients with previous CABG surgery (from a contemporary multicenter US registry). Compared to CTO recanalization of previous CABG surgery, procedural success was $92.5 \%$ in patients without previous CABG surgery. Given the CTO characteristics of enrolled patients in this study, a $97.2 \%$ success rate is a dramatic achievement. The crowbar effect technique showed advantages in treating refractory CTO and improving the success rate of recanalization. Given these factors, the crowbar effect technique may serve as an important alternative method when traditional CTO-PCI fails.

\subsection{About complications of CTO recanalization}

Despite the aggressiveness of these approaches to open an occluded coronary artery, the complication rate is higher than that with non-occluded lesions. The occurrence of coronary perforation in this study was $13.8 \%$. The presence of the blunt stump, severe calcification, severe tortuosity, and occlusion length $>40 \mathrm{~mm}$ are independent predictors of procedural failure with higher complications compared to conventional antegrade wire for CTO.

\subsection{The cause and treatment of coronary perforation}

The causes of coronary perforations in this study were due to stiff guide wires piercing vessel walls. A coronary perforation from a guide wire can usually heal itself and does not require intervention [26]. However, contrast leakage in the vessel from coronary artery perforation can be treated as follows: When a guide wire pierces the vessel wall in small branches or distal branches, the branches can be occluded with some solid special material, such as wire coils, a small piece of thrombus, microspheres of gelatin sponge or a small piece of the patient's subcutaneous fat injected through an microcatheter or a catheter with end-hole [27|28]. If a guide wire perforation has occurred in the proximal vessel or within the CTO, the proximal vessel can also be occluded with these materials. A perforation created by a wire exit within the occluded segment can often be remedied by prolonged balloon inflation and conventional stenting. If that is failure, a covered stent may be used to close the coronary perforation. Sometimes the application of protamine is meaningful for neutralization of heparin during plugging perforation.

\section{References}

[1] Daniel AJ, Roshan W, Krishnaraj R, Jonathan B, Sean G, Charles JK, Akhil K, Ajay KJ, Martin T, Craig AT, Anthony $\mathrm{M}$, Andrew W, Elliot JS. Successful recanalization of chronic total occlusions is associated with improved long-term survival. J Am Coll Cardiol Intv 2012; 5(4): 380-388. 
[2] Dominique J, Jonathan A, Stéphane R. Effectiveness of recanalization of chronic total occlusions: A systematic review and meta-analysis. Am Heart J 2010; 160: 179-187.

[3] Kirschbaum SW, Baks T, Ent M van den, Sianos G, Krestin GP, Serruys PW. Evaluation of left ventricular function, three years after percutaneous recanalization of chronic total coronary occlusions. Am J Cardiol 2008; 101: 179-185.

[4] Spyretta G, Elias AS, George DD. Long-term clinical outcomes after percutaneous coronary intervention for chronic total occlusions. Current Cardiology Reports 2014; 16: 450-461.

[5] Tamburino C, Capranzano P, Capodanno D, Dangas G, Zi MM, Bass TA, Mehran R, Antoniucci D, Colombo A, La MA, Di SME, Stone GW. Percutaneous recanalization of chronic total occlusions: Wherein lies the body of proof? Am Heart J 2013; 165(2): 133-142.

[6] Vijay SR, Craig AT. Coronary chronic total occlusion recanalisation - current techniques and approaches. Interventional Cardiology Review 2013; 8(1): 41-45.

[7] Bufe AG, Haltern, Dinh HW, Wolfertz JH, Schleiting HG. Recanalisation of coronary chronic total occlusions with new techniques including the retrograde approach via collaterals. Neth Heart J 2011; 19(4): 162-167.

[8] Emmanouil SB, Dimitri K, Gerald S.W, James CS, Barry FU, Michael L, Subhash B. Developments in coronary chronic total occlusion percutaneous coronary interventions. 2014 State-Of-The-Art Update. J Invasive Cardiol 2014; 26(6): 261-266.

[9] Emmanouil SB, Dimitri K, Gerald SW, James CS, Barry FU, Michael L, Subhash B. Advances in the management of coronary chronic total occlusions. Journal of Cardiovascular Translational Research 2014; 7(4): 426-436.

[10] Francesco B, Maria DV, Thierry L, Antonella T, Yves L, Carlo T. Radial approach for percutaneous coronary interventions on chronic total occlusions: Technical issues and data review. Catheterization and Cardiovascular Interventions 2014; 83(1): 47-57.

[11] Shah, SA, Shapiro, RJ, Mehta R et al. Impact of enhanced external counterpulsation on canadian cardiovascular society angina class in patients with chronic stable angina: A meta-analysis. Pharmacotherapy 2010; 30: 639-645.

[12] Muhammad FK, Christopher SWMS, Hoang MT, Mohammad RM. Effects of percutaneous revascularization of chronic total occlusions on clinical outcomes: A meta-analysis comparing successful versus failed percutaneous intervention for chronic total occlusion, Catheterization and Cardiovascular Interventions 2013; 82(1): 95-107.

[13] Joren M, Philippe S, Luigi DS, Emanuele B, Joseph D. Chronic total occlusions for intermediate volume operators: An antegrade step-up algorithm allows high success in easy and intermediate difficult CTO lesions. World Journal of Cardiovascular Diseases 2013; 3: 536-542.

[14] Tesfaldet TM, Owen M, Mohammed A, Anna K, George C, Bavan VR, Shuaib A, Jerrold G, Subsash B, Emmanouil SB. Long-term outcomes of successful chronic total occlusion percutaneous coronary interventions using the antegrade and retrograde approach. J Interv Cardiol 2014; 27(5): 465-471.

[15] Kim HY. Percutaneous recanalization of coronary chronic total occlusions: Current devices and specialized wire crossing techniques. Korean Circ J 2010; 40(5): 209-215.

[16] Dimitri K, Nicholas L, Anna K, Harold C, William LL. Development of a high-volume, multiple-operator program for percutaneous chronic total coronary occlusion revascularization: Procedural, clinical, and cost-utilization outcomes. Cathet. Cardiovasc. Intervent 2013; 82(1): 1-8.

[17] Han Y, Wang S, Jing Q, Li Y, Zhang J, Ma Y, Luan B. Percutaneous coronary intervention for chronic total occlusion in 1263 patients: A single-center report. Chinese Medical Journal 2006; 119(14): 1165-1170.

[18] Galassi AR, Tomasello SD, Reifart N, Werner GS, Sianos G, Bonnier H, Sievert H, Ehladad S, Bufe A, Shofer J, Gershlick A, Hildick-Smith D, Escaned J, Erglis A, Sheiban I, Thuesen L. In-hospital outcomes of percutaneous coronary intervention in patients with chronic total occlusion: Insights from the ERCTO (European Registry of Chronic Total Occlusion) registry. Euro Intervention 2011; 7(4): 472-479.

[19] Yoshihiro M, Takeshi K, Yasuhiko H, Toshiya M. In-hospital outcomes of contemporary percutaneous coronary intervention in patients with chronic total occlusion: Insights from the J-CTO registry (multicenter CTO registry in Japan). J Am Coll Cardiol Intv 2010; 3:1 43-151.

[20] Paul F, Merril LK, Asim NC, Galbraith PD, Azriel BO, Sergey Y, Sharon G, Michelle S, Max W, Daniel B, John DS, Graham AW. Current perspectives on coronary chronic total occlusions: The canadian multicenter chronic total occlusions registry. J Am Coll Cardiol 2012; 59(11): 991-997.

[21] Owen M, Vishal GP, Tesfaldet TM, Eric F, Mohammed A, Bavana VR, Shuaib MA, Subhash B, Emmanouil SB. Longterm outcomes with the use of the cross boss and stingray coronary CTO crossing and re-entry devices. J Invasive Cardiol 2013; 25(11): 579-585.

[22] Thompson CA. Percutaneous revascularization of coronary chronic total occlusions. The new era begins. J Am Coll Cardiol Intv 2010; 3(2): 152-154.

[23] David MS, John AH, Barry DR, Steven PM. Success rates of percutaneous coronary intervention for chronic total occlusions and long-term survival in patients with diabetes mellitus. Diab Vasc Dis Res 2006; 3(1): 45-51.

[24] Tesfaldet TM, Owen M, Eric F, Vishal GP, Abdallah ES, Mohammed EA. Application of the "hybrid approach" to chronic total occlusion interventions: A detailed procedural analysis. J Interven Cardiol 2014; 27: 36-43. 
[25] Christopoulos G, Menon RV, Karmpaliotis D, Alaswad K, Lombardi W, Grantham JA, Michael TT. Application of the "hybrid approach" to chronic total occlusions in patients with previous coronary artery bypass graft surgery (from a Contemporary Multicenter US registry). Am J Cardiol 2014; 113(12): 1990-1994.

[26] Daisuke M, Atsunori O, Motoo D, Hiroyuki N, Katsuomi I, Kenshi F. Treatment of collateral channel perforation during percutaneous coronary intervention for chronic total occlusion with retrograde approach. Cardiovascular Intervention and Therapeutics 2014; 29(1): 86-89.

[27] Asouhidou, VK. Successful embolization of iatrogenic ruptured coronary artery using Onyx: A new technique, Acute Cardiac Care 2014; 16: 123-132.

[28] Vishal GP, Kimberly MB, Aracely T, Owen M, Tesfaldet TM, Nathan L, Mohammed A, Deborah S, Daisha C, Shuaib A, Subhash B, Emmanouil SB. Angiographic success and procedural complications in patients undergoing percutaneous coronary chronic total occlusion interventions: A weighted meta-analysis of 18,061 patients from 65 studies. JACC Cardiovasc Interv 2013; 6(2): 128-136. 